\title{
Geographic coordinates guide natural product discovery
}

Secondary metabolites that are encoded by soil bacteria have many ecological roles and, in addition, these compounds have important biomedical applications, for example, as a source of structures for the development of small-molecule therapeutics. Many biomedically important secondary metabolites are synthesized from polyketide synthase (PKS) and non-ribosomal peptide synthetase (NRPS) biosynthetic gene clusters. However, the distribution of such biosynthetic gene clusters in the environment, and the environmental factors that

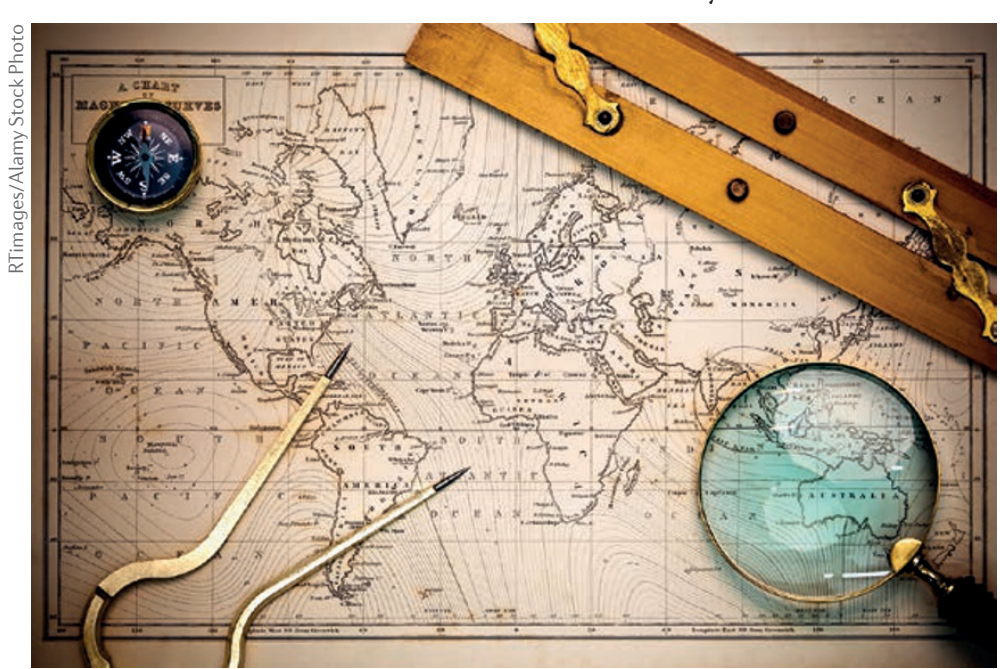

contribute to differences in the composition of those gene clusters found in geographically distant soil microbiomes, are not well understood.

In this study, Lemetre, Maniko et al. set out to systematically identity the environmental factors that are associated with changes in bacterial secondary metabolism across natural environments across the Australian continent. To this end, the authors sequenced PCR amplicons that were generated using primers targeting the NRPS adenylation domain and the PKS ketosynthase domain to

a correlation
between
metagenome
composition
and sample-site
latitude

a correlation

etween composition and sample-site

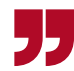

metagenome assess biosynthetic domain composition and richness in soils that were collected at geographically distant sites across the Australian continent. Targeted sequencing of secondary metabolite biosynthesis domains was coupled to associated metadata that were collected for each collection site (that is, latitude, longitude, soil $\mathrm{pH}$, precipitation, altitude, average yearly rainfall and yearly average daily high temperature). Although the authors reported site-specific variations in biosynthetic domain richness, they did not detect any significant correlations between richness and any environmental parameters measured. By contrast, the authors observed a correlation between metagenome composition and sample-site latitude and, to a lesser extent, changes in soil $\mathrm{pH}$. The finding that differences in soil metagenome biosynthetic domain composition correlates with latitude might guide future natural product discovery efforts.

Andrea Du Toit

ORIGINAL ARTICLE Lemetre, C., Maniko, J. et al. Bacterial natural product biosynthetic domain composition in soil correlates with changes in latitude on a continent-wide scale. Proc. Natl Acad. Sci. USA http://dx.doi.org/10.1073/pnas. 1710262114 (2017) 